\title{
Inverted method for fabricating a nano-aperture device with subwavelength structures
}

A. Suutala___Department of Electrical and Information Engineering, Microelectronics and Materials Physics Laboratories, EMPART Research Group of Infotech Oulu, University of Oulu, P.O. Box 4500, FI-90014 Oulu, Finland

J. Olkkonen VTT Technical Research Centre of Finland, Tietotie 3, P.O. Box 1000, 02044 VTT, Espoo, Finland D. C. Cox Advanced Technology Institute, University of Surrey, Guildford, Surrey GU2 7XH, United Kingdom J. Lappalainen and H. Jantunen Department of Electrical and Information Engineering, Microelectronics and Materials Physics Laboratories, EMPART Research Group of Infotech Oulu, University of Oulu, P.O. Box 4500, FI-90014 Oulu, Finland

An inverted method for fabricating a plasmonic nanoaperture device by using focused ion beam (FIB) milling and focused electron beam _FEB_ induced deposition is proposed here. The device structure presented consists of a periodic annulus grating pattern along the interface of a quartz substrate and sputtered aluminum layers and a cylindrical high-index filled nanoaperture through the aluminum film in the center of the grating. FIB milling was used to process the annulus pattern on the quartz substrate. A dielectric nanopost_or inverted nanoaperture_ was fabricated by FEB-induced deposition using tetraethyl orthosilicate as a precursor. The device geometry was characterized by atomic force microscopy and scanning electron microscopy. The structural processability of the device was proven with adequate accuracy and the properties of the materials also met the conditions of the device model in terms of functionality. 


\section{INTRODUCTION}

Subwavelength structures in nanoapertures allow creation of photonic systems beyond the diffraction limit, offering high field enhancements and the advantages of a nanometer scale. In these structures, annular surface corrugations in a metal film around a single, central, subwavelength-sized aperture can greatly enhance otherwise weak transmission through the aperture.1-5 This enhancement is widely attributed to the influence of surface plasmon polaritons, which are surface electromagnetic waves that propagate parallel to a metal/dielectric interface. Excitation of surface plasmons by light is denoted as surface plasmon resonance, whose frequency can be adjusted to a desired value by changing the geometry of the surface pattern.6,7 The main advantage is that in this manner an optical signal can be squeezed into minuscule structures.

Devices that exploit surface plasmon resonance are, e.g., nanoapertures, 8 beam splitters, 9 and nanolenses.10 Nanoapertures are capable of producing a much smaller spot size than is possible with classical optics, and the applications proposed are high-density optical data storage, near-field scanning optical microscopy, and nanolithography.7 In general, single nanoaperture structures3,7 and subwavelength hole arrays11-13 have been fabricated by direct milling on metal thin films.

This article presents an inverted method for fabricating a nanoaperture device with subwavelength structures using focused ion beam _FIB_ milling and focused electron beaminduced _FEB-induced_ deposition together with thin-film technology. The feasibility of the proposed method is evaluated by comparing the dimensions of the fabricated nanoaperture with modeled ones.

\section{MODELING}

Interaction of light with a metallic nanoaperture surrounded by concentric surface corrugations is modeled by the body of revolution finite difference time domain _BORFDTD _ method,14 which assumes that the physical structure to be modeled is cylindrically symmetric. The BOR-FDTD method represents the electric and magnetic field components in cylindrical coordinates ${ }_{-},,_{-}, z_{-}$, where ${ }_{-}$, and $\mathrm{z}$ have their conventional meanings. Due to the fact that the electric and magnetic field components in the cylindrical coordinates are periodic in _, they can be presented as a complex Fourier series,

$$
\mathbf{E}(\rho, \phi, z)=\sum_{m=0}^{\infty} \mathbf{E}_{m}(\rho, z) \exp (j m \phi),
$$


$\mathbf{H}(\rho, \phi, z)=\sum_{m=0}^{\infty} \mathbf{H}_{m}(\rho, z) \exp (j m \phi)$

The complex amplitudes of $\mathbf{E}_{\mathrm{m}_{--}}, \mathrm{z}_{-}$and $\mathbf{H}_{\mathrm{m}_{--}}, \mathrm{z}_{-}$are solved in a two-dimensional mesh, which is a significant advantage compared with the three-dimensional FDTD method. As an additional advantage, the BOR-FDTD method resolves exactly the cylindrical shape of the structure. In the series expansions _ ${ }_{-}$ and $2_{-}$, the mode number $m$ goes from zero to infinity. In practice, the incident field defines the number of modes that must be solved. For example, problems in which the incident field is a linearly polarized plane wave, or a Gaussian beam, or a hybrid HE1n mode of an optical fiber, require only the solution of the mode $\mathrm{m}=1$. On the other hand, a radially polarized Bessel beam or the TM01 mode of an optical fiber requires only the solution of the mode $\mathrm{m}=0$. The computation domain is terminated using a uniaxial perfectly matched layer-absorbing boundary condition, 14 and materials in which the real part of the refractive index is smaller than the imaginary part, as is the case with noble metals at optical frequencies, are modeled with the Lorentz dispersion model based on the auxiliary differential equation.15 To obtain accurate results with the BOR-FDTD method, the two-dimensional BOR-FDTD mesh has to be adequately sampled. In practice, a mesh with at least 30 mesh points per wavelength is required to keep numerical dispersion errors under control.15 In this study, we used a uniform mesh with __ $={ }_{-} \mathrm{z}^{=} \mathrm{r} / 20$, where $\mathrm{r}$ is the radius of the cylindrical hole and ${ }_{--}$and $\mathrm{z}_{\mathrm{z}}$ are the mesh space increments in the _ and $\mathrm{z}$ directions, respectively.

The device structure _Fig. 1_ consists of a periodic annulus corrugation pattern along the interface of a dielectric quartz substrate and a 300_5 nm thick aluminum layer. The cross section of the corrugation pattern is square-wave shaped and it has a period of $600 \mathrm{~nm}$, where the width of each trench and ridge is 300_10 nm. The trenches are 100_5 nm deep. The total number of grooves in the corrugation pattern is defined to be 15 . A cylindrical dielectric nanoaperture is placed in the center of the corrugation pattern and goes through the aluminum film. The aperture has a diameter of $100 \_10 \mathrm{~nm}$ and is supposed to have a higher refractive index than the quartz substrate _n=1.5_ to create a Fabry-Pérot-like resonator structure. The modeling of the device is presented elsewhere in more detail.16 


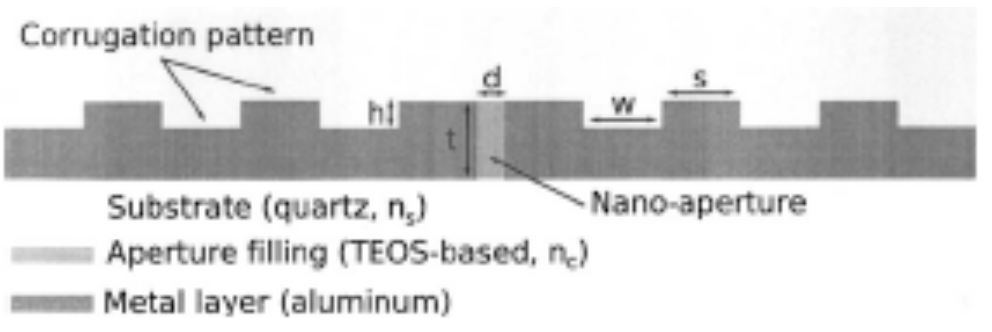

FIG. 1. Cross-sectional image showing the core structure of the device, where $\mathrm{n}_{-} \mathrm{n}$ s and $\mathrm{h}=100 \mathrm{~nm}, \mathrm{~d}=100 \mathrm{~nm}, \mathrm{t}=300 \mathrm{~nm}$, $\mathrm{w}=300 \mathrm{~nm}$, and $\mathrm{s}=300 \mathrm{~nm}$.

\section{EXPERIMENT}

The fabrication process, denoted as an inverted method for fabricating a nanoaperture, contains four main steps:_1_ grounding layer deposition,_2_ FIB milling, _3_ focused electron beam-induced deposition _FEBID_, and _4_ metal layer deposition by sputtering, as shown in Fig. 2. The FIB milling and FEBID experiments were carried out in an FEI Nova 600 NanoLab DualBeam FIB system, which is comprised of a high-resolution field emission scanning electron microscope _FESEM_ and a scanning gallium ion beam column. This machine uses a focused $\mathrm{Ga}^{+}$ion beam with energy of 5-30 keV and a probe current of 1 pA-20 nA. The beam current can be adjusted in 15 steps. The $\mathrm{Ga}^{+}$ion beam resolution is $7 \mathrm{~nm}$ at $1 \mathrm{pA}$ beam current and a fixed $30 \mathrm{keV}$ accelerating voltage. The beam spot size and resolution vary with the ion beam current. Electron optics consists of a high-resolution field emission SEM column. The acceleration voltage can be adjusted continuously from 0.2 to $30 \mathrm{kV}$ and the beam current up to $20 \mathrm{nA}$ in 21 steps. The stage can be moved in the $\mathrm{X}, \mathrm{Y}$, and $\mathrm{Z}$ directions and rotated $360^{\circ}$ and tilted from $-10^{\circ}$ to $60^{\circ}$. During the writing process the stage is stationary, and only the beam scans the sample surface. The ion beam is focused on the sample surface with a normal incident angle. An external pattern writing system, Nanometer Pattern Generation System _NPGS_, is connected to the SEM/FIB machine. The NPGS allow its user to design delineation of complex structures sized from nanometers up to the maximum field of view of the microscope. Process parameters such as beam energy, beam current, dose/dwell time, number of passes/scans, beam overlapping information, magnification, etc., are stored in run files. The samples consist of a 500 _m thick quartz substrate and an approximately $30 \mathrm{~nm}$ thick film of gold _Au_deposited on top. A few nanometers of chrome_Cr_ were deposited on the substrate to improve gold adhesion. The gold layer eliminates surface charges while the samples are exposed to electron and ion beams. Sample charges could eventually drift the beams and deform the shape of the beams during processing and cause unwanted artifacts. The FIB milling process was separated into two stages: _ ${ }_{\text {_ }}$ creating a device window and _ ${ }^{2}$ milling the annulus grating pattern. The device window size was defined to be $25 \_25 \_0.2$ _m 3 . The grating pattern was then milled at the bottom of the device window. Both processes were done with a $30 \mathrm{kV}$ 
acceleration voltage. The fixed beam currents in the device window and the annulus grating milling were 1 $\mathrm{nA}$ and $30 \mathrm{pA}$, respectively. To reach the correct target depths in both structures, a set of patterns was milled using different ion doses.

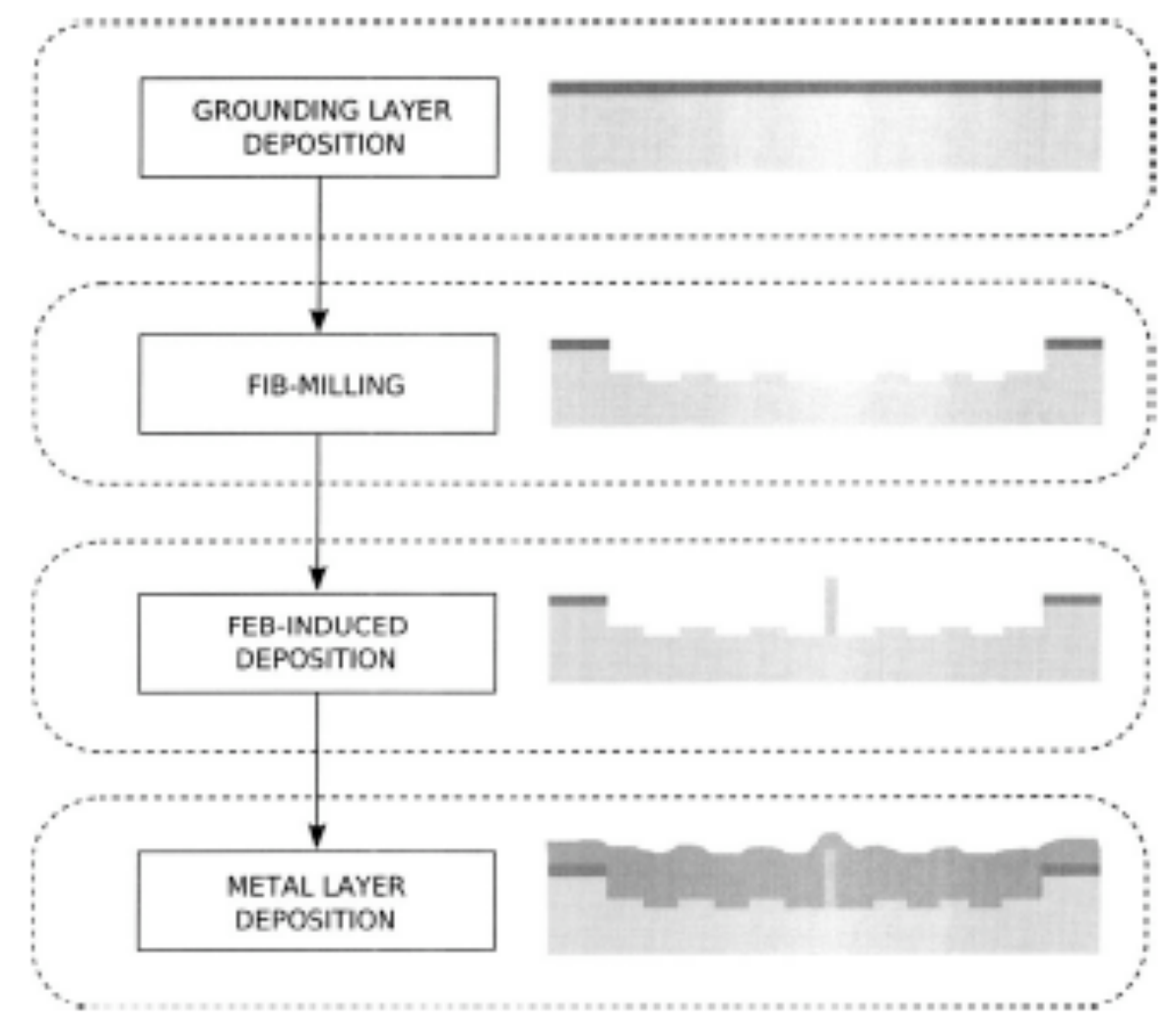

FIG. 2. Process flow showing the main processing steps of the inverted fabrication method.

FIG. 2. Process flow showing the main processing steps of the inverted fabrication method.

After the FIB milling processes, a high-index cylindrical nanopost was deposited in the center of the corrugation pattern using FEBID and tetraethyl orthosilicate _TEOS_ as a precursor. TEOS _Ref. 17_ is a compound of silicon, oxygen, carbon, and hydrogen. Its molecular formula is $\mathrm{SiO}_{4} \mathrm{C}_{8} \mathrm{H}_{20}$ and its molar mass is $208.32 \mathrm{~g} / \mathrm{mol}$. TEOS is a very volatile transparent colorless liquid with a vapor pressure of $2 \mathrm{mbar}$ at $25{ }^{\circ} \mathrm{C}$. TEOS transforms into a solid dielectric material under electron beam irradiation. The chemical composition of the produced material is $\mathrm{Six}_{\mathrm{y}} \mathrm{C}_{z}$, where the normalized $\mathrm{x}: \mathrm{y}: \mathrm{z}$ ratio of the elements is 1:2.45:2.88, respectively. In the deposition process, the focused electron beam is manually aligned with the center of the corrugation pattern. The deposition was done with a so-called spot command program. This means the beam is focused on the same spot during the deposition. When electrons collide with the TEOS gas molecules, a solid material is formed. Since the cross section of the electron beam in the $x-y$ plane is 
circular, the shape of the pillar is cylindrical. Its diameter was controlled by changing the electron beam currents and acceleration voltages, which eventually change the beam spot size. Figure 3 shows the electron beam current as a function of acceleration voltage for each spot. The beam currents were measured using Faraday's cup. In this experiment the temperature of the TEOS was $29^{\circ} \mathrm{C}$. The gas needle was at a $45^{\circ}$ angle and approximately $100 \_\mathrm{m}$ above the sample surface. The base pressure of the chamber was 2_10-6 mbar and it was raised to 6_10-4 mbar after the TEOS was pumped into the chamber. Gas flow was shut down during the deposition process because it was noticed that the continuous flow caused an offset of a few hundreds of nanometers. An external electrostatic beam blanker, Scanservice Beam Blanker model 980, was connected to the system to minimize electron beam tailing effects. Finally, an approximately $600 \mathrm{~nm}$ thick aluminum film was deposited on top of the structure using dc magnetron sputtering in a JLS Designs Ltd. Mini-Lab MPS 500 sputtering system. A vacuum chamber was pumped down with a cryopump and the base pressure was 10-6 Torr. The surface of the target was presputtered before the actual deposition process and the target was covered with a shutter during precleaning. Argon was used as precursor with a flow rate of 25 SCCM _ SCCM denotes cubic centimeter per minute at STP_. During the process, the chamber pressure was maintained at $5 \mathrm{~m}$ Torr using an automatic pressure valve and the plasma current was controlled and monitored to maintain a steady growth rate of the metal films. In this case, the plasma current was $500 \mathrm{~mA}$ and the growth rate was about $10 \mathrm{~nm} / \mathrm{min}$. The structure of the device was analyzed by using a Nova 600 NanoLab FESEM and a Veeco Dimension 3100 Nanoscope IV scanning probe microscope.

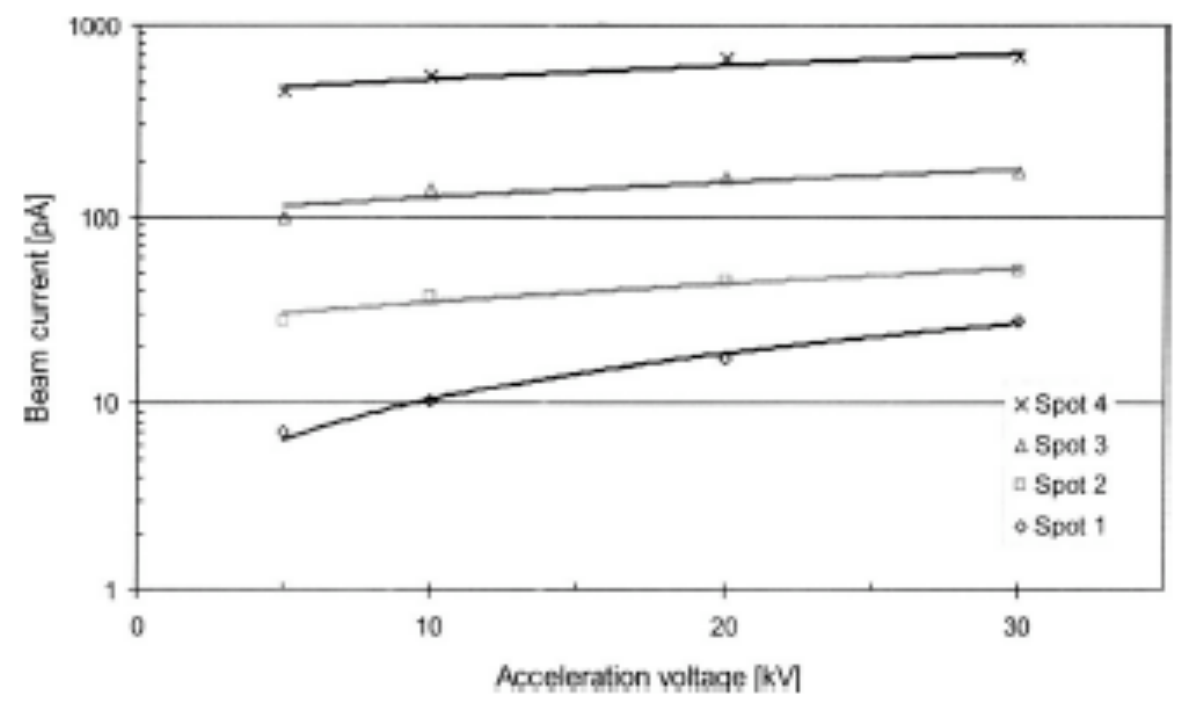

FIG. 3. Electron beam current vs the acceleration voltage in different spot configurations. 


\section{RESULTS AND DISCUSSION}

Atomic force microscopy_AFM_ analyses revealed that a suitable ion dose for a 25_25_0.2_m3 device window in the FIB milling process was 95000 _C/cm2 when using $1 \mathrm{nA}$ beam current, $30 \mathrm{~nm}$ spacing, and 250 repetitive scans over the area. The desired 100_5 nm deep annulus grating pattern was achieved by using an ion dose of $44000 \_\mathrm{C} / \mathrm{cm} 2$ during static FIB milling parameters with a $30 \mathrm{pA}$ beam current, $5 \mathrm{~nm}$ spacing, and 100 scans. In both cases, the high number of repetitive scans were used because it significantly reduced the amount of redeposited material and resulted in smooth surfaces and sharp edges along the corrugation pattern. $R_{q}$ at the bottom of the device window was $0.3 \mathrm{~nm}$. Figure 4 shows the AFM-measured threedimensional_3D_profile of the FIB-milled structures on quartz. With these processing parameters the total milling time was approximately $50 \mathrm{~min}$. The sputtered gold layer completely eliminated surface charges and ion beam drifts.

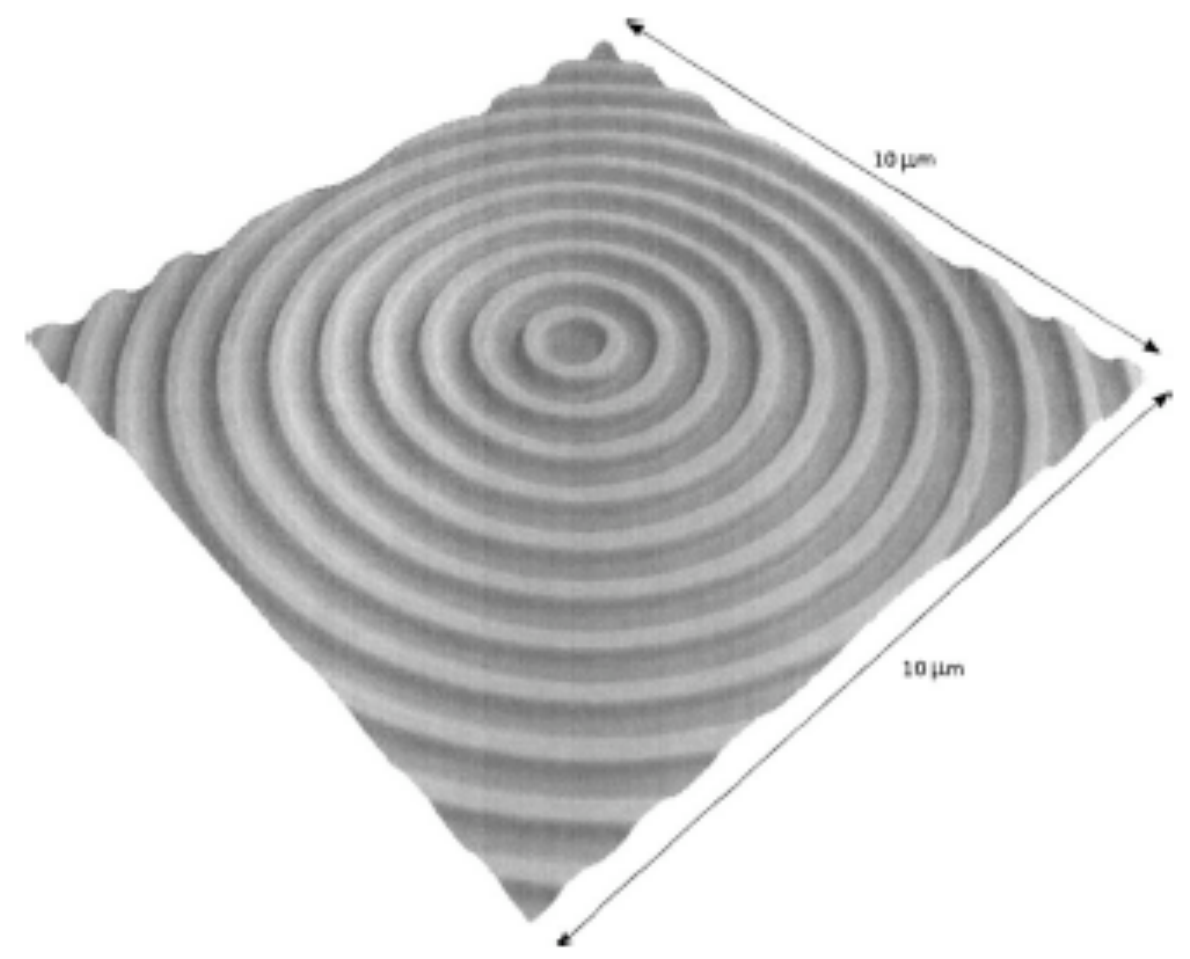

FIG. 4. 3D AFM profile of the FIB-milled corrugation pattern on quartz.

Figure 5 shows the diameters of the FEBID TEOS-based dielectric posts as a function of electron beam spot size. The factor with the most significant effect on the diameter of the deposited posts is electron beam spot size. Less significant factors include acceleration voltage and secondary electron emission during deposition. Figure 6 shows the estimated deposition rate of TEOS-based dielectric material as a function of 
acceleration voltage when using different spot sizes. The estimation is based on calculations of the volumes of the deposited posts from SEM micrographs. Figure 5 shows the diameters of the individual posts achieved in this experiment with different spot sizes and acceleration configurations. For this particular device the stepping scale is adequate. The FEBID technique allows creation of less than $100 \mathrm{~nm}$ diameter posts with a high aspect ratio. However, it must be said that because of the rather large margin of error, the diameters can easily overlap each other on different occasions. The desired post with a 100_10 nm diameter was successfully deposited by spot number 3 using an acceleration voltage of $30 \mathrm{kV}$ and an electron beam current of $0.43 \mathrm{nA}$. The theoretical probe size with a working distance of $10 \mathrm{~mm}$ for spot number 3 in the aforementioned setup was $1 \mathrm{~nm}$. A FESEM micrograph_Fig. 7_a_- shows the deposited nanopost in the center of the FIB-milled corrugation pattern on quartz.

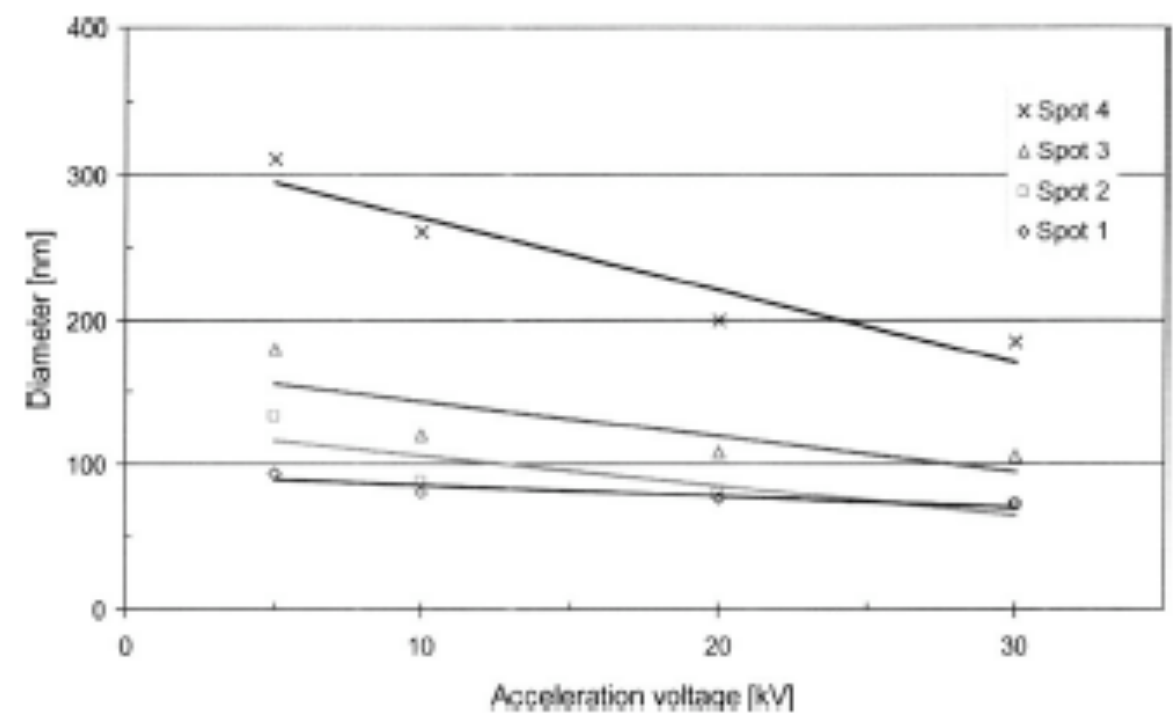

FIG. 5. Diameter of the FEB-induced deposited nanoposts of TEOS vs the acceleration voltage in different spot configurations.

This so-called spot command FEBID process is not quite reliable in terms of repeatability, since it requires precise conditions each and every time: gas pressure, electron beam current, quality of focus, deposition time, quality of vacuum, etc. The value of the electron beam current is significantly affected by the beam apertures, lens alignments, and the age of the electron source. To improve process reliability, the electron beam current should be measured using Faraday's cup and adjusted to the desired value before each deposition. Another problem is the stepping, since the spot size and the beam current are tied to the acceleration voltage. This means it is very difficult to achieve an arbitrary-sized post. 


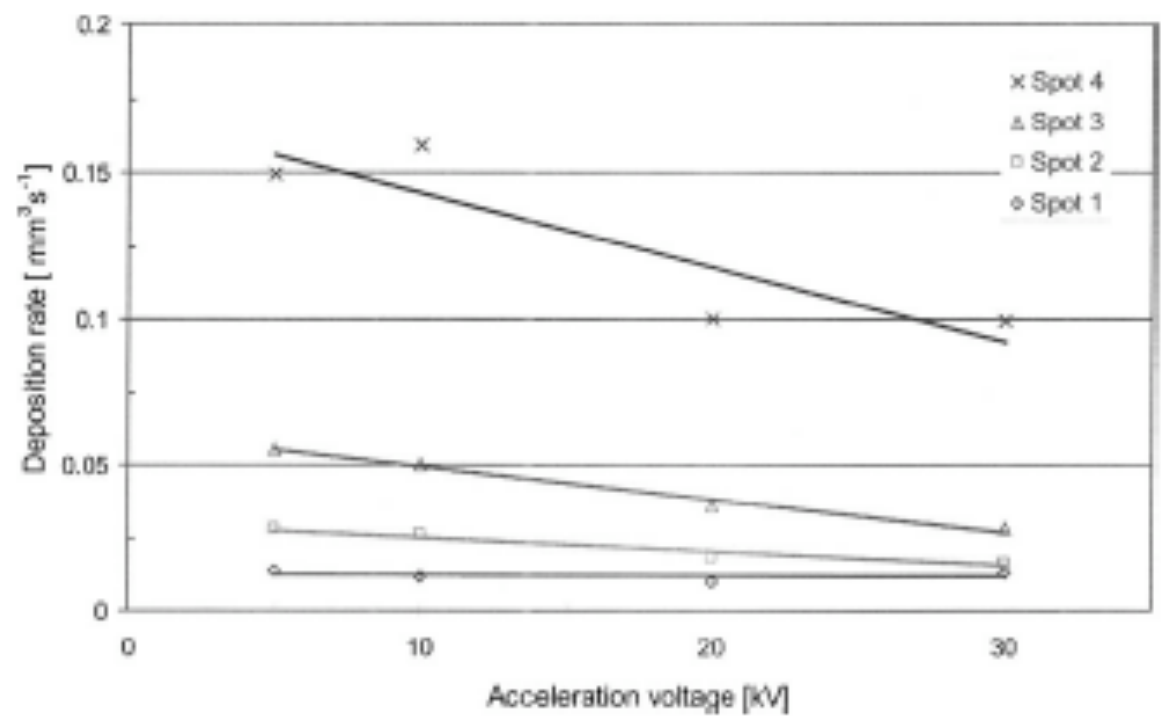

FIG. 6. Deposition rate in the FEBID process.

Figure 7_b_ shows the device structure after aluminum deposition. Aluminum accumulates on the overlong dielectric post and forces it to bend. This casts a shadow along the sample, which causes unwanted nanocavities in the metal layer. This can be seen in the cross-sectional FESEM micrograph _Fig. 7_c_-. Alongside with problems in repeatability, the controlling over the FEBID process needs further development since the height of the deposited dielectric columnar structure was so excessive. Alternatively, FIB milling could be used to modify deposited post before or after metallization to reduce the unwanted effects and get the precise columnar structure without any deformation. This option has been outlined but was not yet implemented here. The micrograph also shows the profile of the quartzaluminum interface and the uniform dielectric nanoaperture.

The aperture is optically transparent in the ultraviolet and visible regions and it has a refractive index slightly higher than that of the quartz substrate. The structure generates a Fabry-Pérot-like resonator because of inequality in the refractive indices, which based on the simulation results is supposed to enhance the performance of the device. 

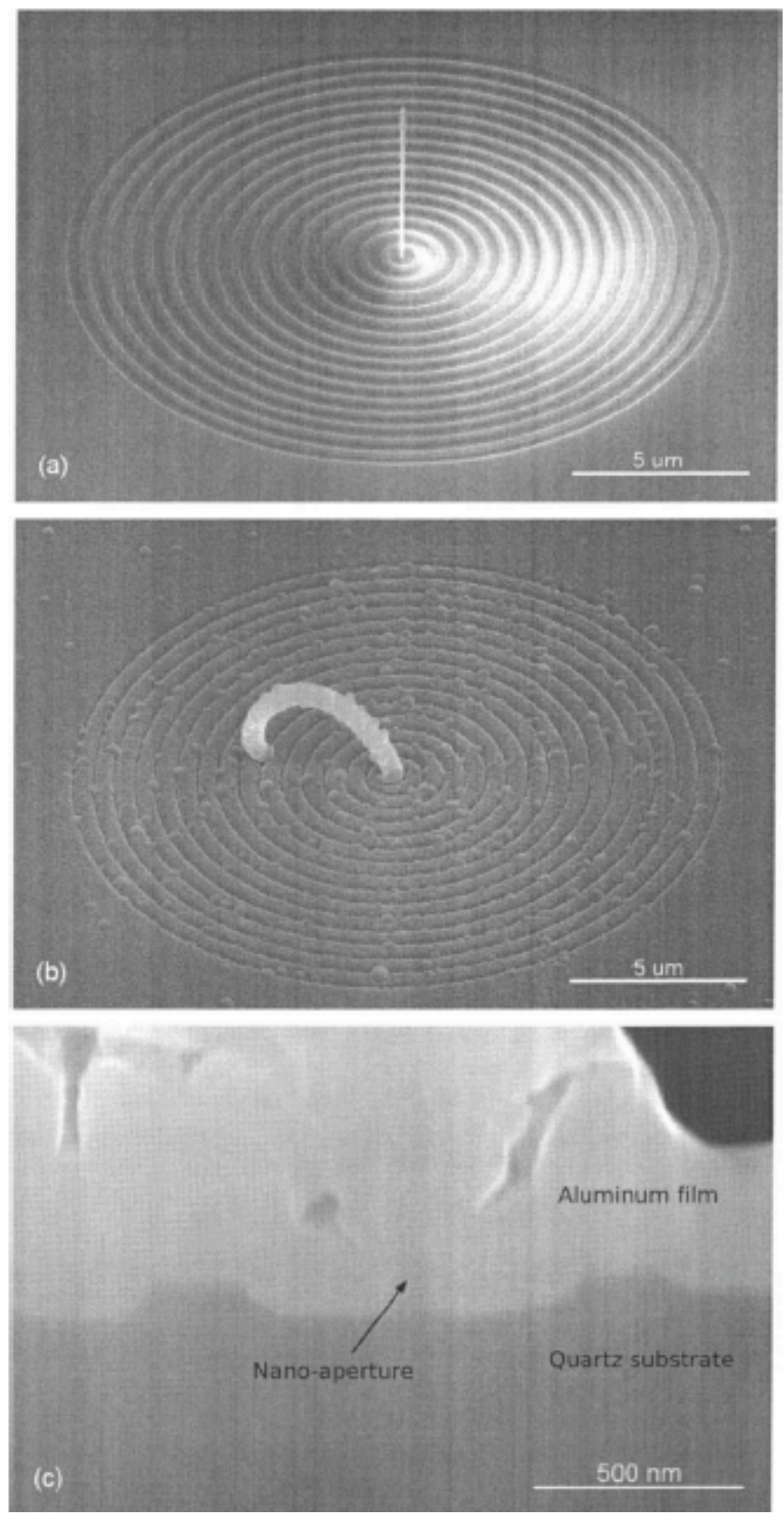

FIG. 7. FESEM micrographs showing the deposited nanopost in the center of the FIB-milled corrugation pattern on quartz _a_, device structure after aluminum deposition _ $b_{-}$, and the cross section of the final structure _c_. 
The aforementioned processes and techniques proved the processability of the core structure of the device with adequate accuracy by fulfilling the geometrical and materialbased demands set by the modeling. FIB milling on the quartz substrate eliminated milling of rather difficult metal films. Added to this, the dielectric-metal interface can be produced by metallization using whatsoever metal film is compatible with the quartz substrate without a need to reoptimize the FIB milling parameters. This allows the use of metal films that are impossible to pattern in the nanometer scale.

Gallium contamination and the defects it causes limit processing and analysis accuracy alike. Even if the geometrical dimensions were attainable, the functional properties of the device may in many respects depend on gallium contamination18 and its alternations, especially in the substrate's optical properties. Gallium occurrences in the processed area are mainly caused by contaminated redeposited material. Neutral particles generated in the ion column may also play a key role in contamination. For all intents and purposes, defects are always a part of FIB processing. Therefore, contamination may be crucial in nanoscale structures. A mechanical shutter could eliminate neutral particles and processing precise areas with smaller ion energy could reduce defects. A gas-assisted postcleaning process could be used to reduce gallium-contaminated redeposited material.

\section{SUMMARY}

A fabrication process for a nanoaperture device with a subwavelength structure was carried out using an inverted method. The results showed that the proposed fabrication enabled the processability of the core structure of the device by fulfilling the geometrical and material-based demands set by the modeling. Trenches and ridges in the FIB-milled corrugation pattern were measured to be 300_10 nm in width and 100_10 nm in depth. The FEB-induced deposited nanoaperture of TEOS had a diameter of 100_10 nm. The FIB technique was used to fabricate the periodic annulus structure in the dielectric quartz substrate layer with adequate resolution. A cylindrical dielectric nanoaperture with a diameter of about $100 \mathrm{~nm}$ was successfully fabricated using focused electron beam-induced deposition with TEOS as precursor. The results presented here clearly show that the focused ion beam and electron beam techniques together with thin-film technology can be used to fabricate versatile local nanoscale structures. Development of the device will be continued into a prototype stage.

\section{ACKNOWLEDGMENTS}

This research work was part of the NAPERO _development of novel fabrication methods for nanoscale photonics and microelectronics components_ project, funded by TEKES-National Technology Agency of 
Finland and "The Research and Development Project on Nanophotonics," funded by the Ministry of Education_OPM_. The experiments were carried out in the Advanced Technology Institute_ATI_, University of Surrey, United Kingdom.

1D. E. Grupp, H. J. Lezec, T. Thio, and T. W. Ebbesen, Adv. Mater. _Weinheim, Ger._ 11, 860 _ ${ }^{1999 \_. ~}$

${ }_{2}$ T. Thio, K. M. Pellerin, R. A. Linke, H. J. Lezec, and T. W. Ebbesen, Opt. Lett. 26, 1972 _2001_.

3T. Thio, H. J. Lezec, T. W. Ebbesen, K. M. Pellerin, G. D. Lewen, A. Nahata, and R. A. Linke, Nanotechnology 13, 429_2002_.

4A. Degiron and T. W. Ebbesen, Opt. Express 12, 3694_2004_.

${ }_{5}$ H. J. Lezec and T. Thio, Opt. Express 12, 3629 _2004_.

${ }_{6}$ W. L. Barnes, A. Dereux, and T. W. Ebbesen, Nature _London_ 424, 824 _2003_.

${ }_{7}$ H. J. Lezec, A. Degiron, E. Devaux, R. A. Linke, L. Martin-Moreno, F. J. Garcia-Vidal, and T. W. Ebbesen, Science 297, 820 -2002_.

8T. W. Ebbesen, H. J. Lezec, H. F. Ghaemi, T. Thio, and P. A. Wolff, Nature _London_ 391, 667 _ $1998 \_$.

${ }_{9}$ H. Ditlbacher, J. R. Krenn, G. Schider, A. Leitner, and F. R. Aussenegg, Appl. Phys. Lett. 81, 1762 _2002_.

10Z. Sun and H. K. Kim, Appl. Phys. Lett. 85, 642 _2004_.

11A. Krishnan, T. Thio, T. J. Kim, H. J. Lezec, T. W. Ebbsen, P. A. Wolff, J. Pendry L. Martin-Moreno, and F. J. Garcia-Vidal, Opt. Commun. 200, 1_2001_.

${ }_{12}$ D. E. Grupp, H. J. Lezec, T. W. Ebbesen, K. M. Pellerin, and T. Thio, Appl. Phys. Lett. 77, 1569 _2000_.

13Y. K. Kim, A. J. Danner, J. J. Raftery, Jr., and K. D. Choquette, IEEE J. Sel. Top. Quantum Electron. 11, 1292 _2005_•

14A. Taflove and S. C. Hagness, Computational Electrodynamics: The Finite Difference Time Domain Method, 2nd ed._Artech

House, Boston,

${ }_{15}$ M. Okoniewski, M. Mrozowski, and M. A. Stuchly, IEEE Microw. Guid. Wave Lett. 7, 121 _ 1997 .

16J. Olkkonen, K. Kataja, and D. G. Howe, Opt. Express 14, 11506 _2006_.

17A. Perentes, A. Bachmann, M. Leutenegger, I. Utke, C. Sandu, and P. Hoffmann, Microelectron. Eng. 73-74, 412 _2004_.

${ }_{18}$ C. Lehrer, L. Frey, S. Petersen, M. Mizutani, M. Takai, and H. Ryssel, IEEE Proceedings on Ion Implantation Technology 2000,

Alpbach, Austria,

17-22 September 2000_IEEE, Piscataway, NJ, 2000_, pp. 695-698. 